\title{
Synthesis of UiO-66 Using Solvothermal Method at High Temperature
}

\author{
Ika Diah Rahmawati ${ }^{1}$, Ratna Ediati, and Didik Prasetyoko
}

\begin{abstract}
UiO-66 solids have been synthesized using solvothermal method in which reaction mixtures of zirconium tetrachloride $\left(\mathrm{ZrCl}_{4}\right)$ and 1,4-benzenedicarboxylic acid (BDC) in $\mathrm{N}-\mathrm{N}^{\prime}$-dimethylformamide (DMF) were heated at $140{ }^{\circ} \mathrm{C}$ for $6,12,24,36,48,72$ and 144 hours, respectively. The weight of reaction products, in the form white powder, increased with the increase in heating time up to 72 hours, then decreased. Characterization results using XRD showed that the diffractogram of the solid obtained at heating time of $6 \mathrm{~h}$ had the same pattern to that of reported UiO-66, characterized by a main peak with a high intensity at $2 \theta$ of $7.3^{\circ}$, as well as other characteristic peaks with lower intensity at $2 \theta$ of $8.4^{\circ} ; 25.6^{\circ}$ and $30.6^{\circ}$. The longer the heating time (12 and $\left.24 \mathrm{~h}\right)$, the lower the intensity of the main peak. When the reaction mixtures were heated for $36 \mathrm{~h}$ or longer, the obtained solid diffractograms showed that the main peak intensity at $2 \theta$ of $7.3^{\circ}$ was lower than the second peak, and new peaks appeared at $2 \theta$ of $13.8^{\circ} ; 1^{\circ} .8^{\circ}$ and $17.5^{\circ}$. SEM micrographs showed that the solid synthesized for $6 \mathrm{~h}$ was in the form of clusters of square morphology, whereas solids synthesized for $72 \mathrm{~h}$ showed needle-like surface morphology.
\end{abstract}

Keywords-UiO-66, solvothermal, heating times, XR.

\section{INTRODUCTION}

$\mathrm{M}$ etal organic frameworks (MOFs) have emerged as a new type of functional materials and can be constructed by an infinite number of metal/ligand combinations[1]. Thus have far greater diversity in structures, functional groups, and pore sizes and shapes such as Isoreticular Metal Organic Framework (IRMOF), Zeolite-Imidazolate Framework (ZIF), Hongkong University of Science and Technology (HKUST), etc. The ability of coordination between the metal ions with different linker in MOF frameworks system will generate high enough to control the properties of the resulting MOF. Therefore, this is one factor that allows the MOF can be designed in accordance with the desired properties and applications [2].

Some applications of MOF has been widely reported, such as a hydrogen storage, the separation of gas molecules, and catalysts. Based on some research on the application of the MOF, the MOF has generally some type of thermal stability between $250-400^{\circ} \mathrm{C}$ and chemical stability are quite low [3]. For example, MOF5, shows the thermal stability temperature up to $300^{\circ} \mathrm{C}$ [4] while the HKUST-1 shows the thermal stability at temperatures of $280^{\circ} \mathrm{C}$ [5]. MOF framework stability is controlled by the bonding between inorganic metal (as inorganic brick) and the strength of chemical bonds have determined by the strength of between the inorganic brick and linker [3]. Cavka et al. (2008) reported the synthesis of a new type of MOF using zirconium $(\mathrm{Zr})$ as a building brick and 1,4-benzendikarboksilat acid (BDC) as the organic linker and denoted as UiO-66 (University of Oslo-66) has achieve thermal stability temperature of $540^{\circ} \mathrm{C}$ and chemical stability are quite good as the water solvent, acetone, benzene, and DMF [3]. UiO-66 is synthesized by solvotermal method with a reaction temperature of $120^{\circ} \mathrm{C}$ for 24 hours and $\mathrm{N}, \mathrm{N}^{\prime}$ dimethylformamide (DMF) as the solvent. The resulting

${ }^{1}$ Ika Diah Rahmawati, Ratna Ediati, and Didik Prasetyoko are with Department of Chemical Engineering, Faculty of Industry Engineering, Institut Teknologi Sepuluh November, Surabaya, 60111, Indonesia. surface area of UiO-66 at $1187 \mathrm{~m} 2 / \mathrm{g}$ and morphology of cubic intergrown crystals of UiO-66.

In general, the use of methods of synthesis and the synthesis conditions such as, temperature, time, $\mathrm{pH}$, and the composition of the reactants (metal: ligand) are different influences the structure and characteristics of the resulting MOF. The higher reaction temperature is used then the time required for crystallization of a material more quickly. Research on the effects of temperature and time of solvothermal methode toward to the characteristics of the resulting material has been done on the synthesis of MOF ZIF-8 type. Formation of ZIF-8 crystals achieve optimum crystallinity after the reaction lasted for 24 hours. Increased reaction time is longer does not provide changes to the crystal structure of ZIF-8 [6].

In terms of the use of solvents DMF, a preliminary test we have done to synthesize UiO-66 using the method solvotermal indicate that the composition of the aqueous reactants and solvents [3 and 7] produce different crystallinity by XRD diffractogram pattern. In addition, the use of temperature and long heating time when synthesis also affects the crystallization process. When synthesis at high temperatures, the crystallization process requires a short amount of time. In contrast, if the synthesis is done at lower temperatures the crystallization takes longer.

Generally, UiO-66 is synthesized by several researchers at a temperature of $120{ }^{\circ} \mathrm{C}[3,7,8,9,10,11,12]$. Therefore, in this research, observation of the crystallization process UiO-66 material at higher temperature is $140 \mathrm{oC}$ at some specific reaction time and use of reactants and solvent composition between Cavka, et al., [3] and Abid, et al. , [7] to obtain a good degree of crystallinity and rapid synthesis time.

\section{METHOD}

\section{A. Synthesis of Zr-MOFs}

All chemicals including DMF (Dimethylformamide, Merck, 99\%), Zirconium tetracloride (ZrCl4, SigmaAldrich, 99,0\%), BDC (Benzene-1,4-dicarboxylic acid, Sigma-Aldrich, 98.9\%), chloroform .(CHCl3, Merck, $99,9 \%)$. 
UiO-66 was synthesized following the procedure reported by Cavka et al. [3] and Abid et al. [7] by implementing the solvotermal method at a temperature of $140^{\circ} \mathrm{C}$ with variation of heating times at $6,12,24,36$, 48,72 , and 144 hours.

The synthesis of UiO-66 has been carried out by dissolving $1,5 \mathrm{mmol}$ of Zirconium tetraclorida $(\mathrm{ZrCl} 4)$ and 1,5 mmol Benzene-1,4-dicarboxylic acid (BDC) in $30 \mathrm{~mL}$ Dimethylformamide (DMF), then stirred with magnetic stirrer for 30 minutes. The reaction mixture was then heated in an oven at $140{ }^{\circ} \mathrm{C}$. The reaction mixture was removed from the oven at intervals of 6,12 , $24,36,48,72$ and 144 hours, allowed to cool at room temperature. The solvent was then removed from the vial bottles by decantation, the solid was washed by adding $30 \mathrm{~mL}$ of $\mathrm{DMF}$ and left for 24 hours. The washing process was repeated twice with chloroform as the solvent. The obtained solid was then dried under vacuum at $60-70{ }^{\circ} \mathrm{C}$ and stored in a container with a nitrogen atmosphere and weighed.

\section{B. Characterizations of UiO-66}

$\mathrm{X}$-ray powder diffraction patterns were obtained with an X-ray diffractometer (D8 Advance-Bruker aXS) using $\mathrm{Cu} \mathrm{Ka}$ radiation $(\mathrm{k}=1.5406 \AA)$, with accelerating voltage and current of $40 \mathrm{kV}$ and $40 \mathrm{~mA}$, respectively. SEM analysis (Zeiss EVO MA 10) was used to capture and determine the morphologies of the crystalline UiO66 samples. Thermal stability of UiO-66 was checked by a thermogravimetric analysis (TGA) instrument (TGA/DSC1 STARe system-METTLER TOLEDO). UiO-66 samples were loaded into a pan and heated to $1173 \mathrm{~K}$ at a rate of $5 \mathrm{~K} / \mathrm{min}$. The air gas flow rate was maintained at $10 \mathrm{~mL} / \mathrm{min}$.

\section{RESULT AND DISCUSSION}

\section{A. Synthesis of UiO-66}

Synthesis of UiO-66 is using solvotermal methods in the oven at $140^{\circ} \mathrm{C}$ with a variation of heating time at 6 , $12,24,36,48,72$, and 144 hours to give a solution with two layers of white solid on the bottom and the clear solution at the top. After the heating process, the solution obtained by decantation and the solid washed with DMF immersed to remove residual unreacted. In addition to DMF, the solid washed well with chloroform to remove residual DMF and BDC within the framework of UiO-66 $[7,8]$. The solids were then dried at $60-70^{\circ} \mathrm{C}$ for several hours to evaporate the chloroform which has a boiling point of $61,2^{\circ} \mathrm{C}$. Solids synthesized UiO-66 with long heating time variation of 6-144 hours at a temperature of $140^{\circ} \mathrm{C}$ warming denoted by UiO-66 140C, 6h (a), UiO66 140C, 12h (b), UiO-66 140C, 24h (c), UiO-66 140C, 36h (d), UiO-66 140C, 48H (e), UiO-66 140C, 72h (f), and $\mathrm{UiO}-66140 \mathrm{C}, 144 \mathrm{~h}(\mathrm{~g})$, where the numbers indicate temperature and long heating time. All solids were weighed as crystalline masses of UiO-66. The mass of each crystal with a long heating time is presented in Figure 1.

Weight of changes in the crystal increases with the length of heating time at $140^{\circ} \mathrm{C}$. Weight gain with long heating krital $6,12,24,36,48,72$, and 144 hours respectively $0.4448 ; 0.4503 ; 0.4760 ; 0.4780 ; 0.5015$; 0.5458 , and 0.4701 grams. Weight change of the crystal increases with the length of hearung time at $140^{\circ} \mathrm{C}$. Weight gain of crystal with heating time at $6,12,24,36$,
48, 72, and 144 hours respectively $0.4448 ; 0.4503$; $0.4760 ; 0.4780 ; 0.5015 ; 0.5458$, and 0.4701 grams. The optimum weight occurs at long heating time of 72 hours at 0.5458 grams and the weight loss crystals on a longer heating time of 144 hours.

\section{B. The results of Characterization by X-Ray Diffraction (XRD)}

XRD pattern of UiO-66 at $140^{\circ} \mathrm{C}$ with a variation in heating time up to 144 hours resulted in major peaks and some peaks characteristic varied in intensity as shown in Figure 2. Based on XRD results, it can be seen that the intensity generated by each of the main peak and peak characteristics of UiO-66. The peak intensity of XRD pattern can be obtained about information the degree of crystallinity of $\mathrm{UiO}-66$, especially at peak with $2 \theta$ of $7,3^{\circ}, 8,5^{\circ}$ and $25,6^{\circ}$. The area under the curve of $7,3^{\circ}$, $8,5^{\circ}$ and $25,6^{\circ}$ at $\mathrm{UiO}-66$ with a 6 -hour of heating time has the most extensive area. Therefore, it is assumed that the UiO-66 is synthesized by solvotermal method in $\mathrm{DMF}$ solvent at $140^{\circ} \mathrm{C}$ for 6 hour have a higher degree of crystallinity that is best used as a comparison standard and crystallinity is considered $100 \%$ [6]. The results of UiO-66 crystallinity level are presented in Table 1.

XRD results of UiO- 66 has a main peak at $2 \theta$ angles of $7.3^{\circ}$ and some characteristic peaks at $2 \theta$ of $8.4^{\circ} ; 25.6^{\circ}$, and $30.6^{\circ}[3,14]$ as shown in Figure 2. UiO-66 is formed rapidly on heating time 6 hours produces diffractogram $\mathrm{UiO}-66$ main peaks at $7,4^{\circ}$ and some characteristic peaks at $8,5^{\circ} ; 25,7^{\circ}$, and $30,7^{\circ}$ with the intensity pattern in accordance with the UiO-66 references [3] and simulations [14]. At the heating time of 12 and 24 hours, the intensity of the main peak and peak characteristics lower than 6 hours of heating time. At the heating time of 24 hour, the intensity of the main peak decreased while the characteristic peaks at $8,5^{\circ}$ to $8,7^{\circ}$ with intensity slightly higher than the heating time 12 hours. Indicated that heating time of 24 hours, UiO-66 is synthesized has begun to experience a phase change due to a shift in the two characteristic peaks $\left(8,5^{\circ}\right.$ to $\left.8,7^{\circ}\right)$.

When a longer heating time is 36 to 144 hours, a change in the pattern and intensity of the main peak and the characteristic peaks and appeared several new peaks. When the heating time is 36 hours, decline in the intensity of the main peak and an increase in the characteristic peaks at $8,5^{\circ}$ and appeared several new peaks at $14.8^{\circ}, 15,2^{\circ}, 15.8^{\circ}$, and $17.4^{\circ}$. The longer a heating time is used from $36,48,72$, up to 144 hours , decrease and increase in the intensity of the main and characterictic peak intensity at $7,4^{\circ}$ and $8.5^{\circ}$ of UiO-66. In addition to changes in the XRD pattern and intensity of the main peaks characteristic peaks, new peaks generated also increased along with the length of heating time of synthesis. The emergence of several new peaks indicated that UiO-66 has experienced a phase change. This statement is supported by the results of the characterization of SEM to observe the surface morphology of UiO-66 that had been synthesized.

Weight of form of crystals synthesized at each time synthesis increased weight of along with a heating time of 6 to 144 hours and there is an optimum weight of crystals on a heating time of 72 hours. Based on the results of XRD pattern of UiO-66 that the 6 hour long heating time is the best time to create the product $\mathrm{UiO}$ 66 is synthesized at $140{ }^{\circ} \mathrm{C}$ was 0,4448 grams. 
Crystalline mass at 6 hours heating time is not an optimum weight, but obtained a diffraktogram with XRD pattern and appear peaks corresponding to the literature [3] and simulations [14]. At 6 hours long heating time can be said to have a good crystallinity among all XRD patterns and because it has the highest crystallinity similar to UiO-66 standard.

Generally, UiO-66 synthesized at $120^{\circ} \mathrm{C}$ for 24 hours. The less time is needed to produce UiO-66 at a temperature of $140{ }^{\circ} \mathrm{C}$ is due to the higher temperatures used, the faster the time formation of a crystall. Weight optimum synthesis of UiO-66 at $140{ }^{\circ} \mathrm{C}$ occurs at heating time for 72 hours at 0.5458 grams, but the longer heating time is not selected as the optimum time for the synthesis of UiO-66 at $140{ }^{\circ} \mathrm{C}$, although have the highest weight crystals, there is changes in the pattern of intensity, peak shift characteristics, and some new peaks appear that are not in accordance with the standards UiO-66.

\section{Results Characterization with Scanning Electron Microscopy (SEM)}

Characterization using Scanning Electron Energy Microscophy (SEM) aims to determine the surface morphology UiO-66. The surface morphology of UiO-66 can be seen in Figure 3.

Results from SEM characterization in Figure 3 (a) is UiO-66 are synthesized during the 6-hour heating produces a cubic intergrown crystals. This is consistent with the results of XRD diffractogram (Figure 2 (a)), where the results of the XRD patterns and characteristics of major peaks corresponding to the literature and simulation as well as produce a good crystallinity. Figure

3 (b) is the SEM results at a heating time 72 hours resulted in morphological UiO-66 as needles-like (not cubic integrown). Morphological changes of UiO-66 on heating time at 72 hours is consistent with the XRD results UiO-66 in Figure 2 (f), where is a change in the XRD pattern of the main peak intensity and peak characteristics and some characteristic new peaks appear. Although the resulting optimum weight occurs on heating time 72 hours, but the results of crystal characterization by SEM and XRD diffractogram is not in accordance with the literature and simulation of UiO66. Therefore, the heating time of 72 hours is not selected as an optimum synthesis time UiO-66 at a $140^{\circ} \mathrm{C}$ so that the best time synthesis chosen for the synthesis of UiO-66 is 6 hours due to the results of SEM and XRD diffractogram according to the literature and simulation of UiO-66.

To determine content of the elements in UiO-66, analysis using SEM-EDX with shooting at one point or spot samples as in Figure 4. Analyzed UiO-66 is synthesized sample at the optimum heating time is 6 hours. The output of the analysis by SEM-EDX elemental form of a percentage. Detected elements are carbon $(\mathrm{C})$, oxygen $(\mathrm{O})$, zirconium $(\mathrm{Zr})$, and chlorine (Cl). At UiO-66, the composition of $\mathrm{C}, \mathrm{O}, \mathrm{Zr}$, and $\mathrm{Cl}$, respectively, are $34,57 \%, 29,72 \%, 33,59 \%$, and $02,12 \%$.

\section{Thermal Stability of UiO-66}

Thermal stability analysis of UiO-66 was conducted using a TGA. Weight loss profiles and weight loss rate are presented in Fig. 5. It is clear that three-stage weight loss occurred on UiO-66. The first weight loss appearing before $373 \mathrm{~K}$ is due to the evaporation of surface adsorbed water on $\mathrm{UiO}-66$. The second weight loss at $424-464 \mathrm{~K}$ is attributed to the decomposition of DMF, which was not exchanged by chloroform. The third stage of weight loss at $799-846 \mathrm{~K}$ is ascribed to the decomposition of UiO-66 (Zr-MOFs) to $\mathrm{ZrO} 2$. Abid et al., [7] reported that the thermal stability of UiO-66 was up to $753 \mathrm{~K}$, which is much less than the one in this investigation. This suggests that solvent exchange activation removed more DMF and nonbonding BDC, resulting in higher thermal stability of $\mathrm{Zr}-\mathrm{MOF}$ at $826 \mathrm{~K}$

\section{CONCLUSION}

This research has shown that solvothermal synthesis of UiO-66 with $140^{\circ} \mathrm{C}$, obtained the best heating time at 6 hour and thermal stability up to $826 \mathrm{~K}$. It is based on the result of XRD pattern and SEM image are similar to the standard of UiO-66. Solvothermal synthesis of UiO-66 at $140^{\circ} \mathrm{C}$ with heating time of 36 and 72 hour was not chosen because the result of XRD pattern and SEM image is different with standard of UiO-66, although the optimum weight obtained at 72 hour.

\section{ACKNOWLEDGEMENT}

The authors would like to thank Directorate of Higher Education (DIKTI) in The Ministry of Education and Culture, Laboratory Materials and Energy of Chemistry in faculty of mathematics and science (MIPA), and laboratory material characterization of materials and metallurgical engineering department of industrial technology faculty (FTI) for their cooperation in this research.

\section{REFERENCES}

[1] K. Sumida and J. Arnold, "Preparation, Characterization, and Postsynthetic Modification of Metal-Organic Frameworks: Sythentic Experiments for an Undergraduate Laboratory Course in Inorganic Chemistry," Journal of Chemical Education, no. 88, pp. 92-94, 2011.

[2] L. Ma and W. Lin, "Designing Metal-Organic Frameworks for Catalytic Applications," Top Curr Chem, no. 293, pp. 175-205, 2010.

[3] M. Eddaoudi, D. B. Moler, H. Li, B. Chen, T. M. Reineke, M O'keeffe and O. M. Yaghi, "Modular Chemistry: Secondary Building Units as a Basis for the Design of Highly Porous and Robust Metal-Organic Carboxylate Frameworks," Accounts of Chemical Research, no. 34, pp. 319-330, 2001.

[4] J. Cavka, S. Jakobsen, U. Olsbye, N. Guillou, C. Lamberti, S Bordiga and K. Lillerud, "A New Zirconium Inorganic Building Brick Forming Metal Organic Frameworks with Exceptional Stability," Journal. American. Chemistry. Society.,, no. 130, p. 13850-13851, 2008.

[5] S. R. Venna, J. B. Jasinki and M. A. Carreon, "Structural Evolution of Zeolitic Imidazolate Framework-8," Journal. American. Chemistry. Society, no. 132, pp. 18030-18033, 2010.

[6] H. R. Abid, G. H. Pham, H. M. Ang, S. Wang and M. O. Tade, "Nanosize Zr-metal organic framework (UiO-66) for hydrogen and carbon dioxide storage," Chemical Engineering Journal, no. 187, p. 415-420, 2012.

[7] H. R. Abid, G. H. Pham , H. M. Ang, M. O. Tade and S. Wang, "Adsorption of $\mathrm{CH} 4$ and $\mathrm{CO} 2$ on $\mathrm{Zr}$-metal organic frameworks," Journal of Colloid and Interface Science, no. 366, p. 120-124, 2012.

[8] A. M. Ebrahim, B. Levasseur and T. J. Bandosz, "Interactions of $\mathrm{NO} 2$ with Zr-Based MOF: Effects of the Size of Organic Linkers on NO2 Adsorption at Ambient Conditions," Langmuir, no. 29, p. 168-174, 2013.

[9] O. G. Nik, X. Y. Chen and S. Kaliaguine, "Functionalized metal organic framework-polyimide mixed matrix membranes for 
CO2/CH4 separation," Journal of Membrane Science, no. 413414, p. 48-61, 2012.

[10] J. Kim, S.-N. Kim, H.-G. Jang, G. Seo and W.-S. Ahn, "CO2 cycloaddition of styrene oxide over MOF catalysts," Applied Catalysis A: General, no. 453, p. 175-180, 2013.

[11] P. M. Schoenecker, C. G. Carson, H. Jasuja, C. J. J. flemming and K. S. Walton, "Effect of Water Adsorption on Retention of Structure and Surface Area of Metal-Organic Frameworks".

[12] J. Li, S. Cheng, Q. Zhao, P. Long and J. Dong, "Synthesis and hydrogen-storage behavior of metal-organic framework MOF5," International Journal of Hydrogen Energy, no. 132, pp. 18030-18033, 2010.

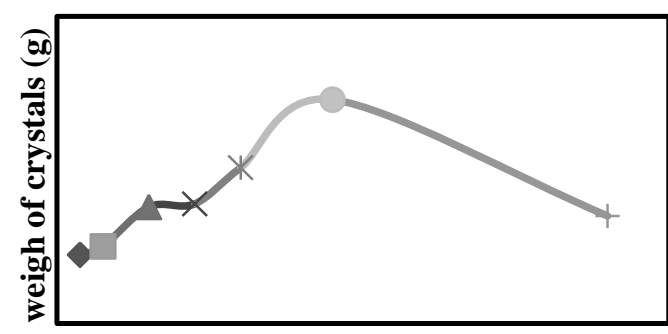

heating time (h)

Figure 1. Corelation Between Weight of Crystal and Heating Time of $\mathrm{UiO}-66$ at $140^{\circ} \mathrm{C}$

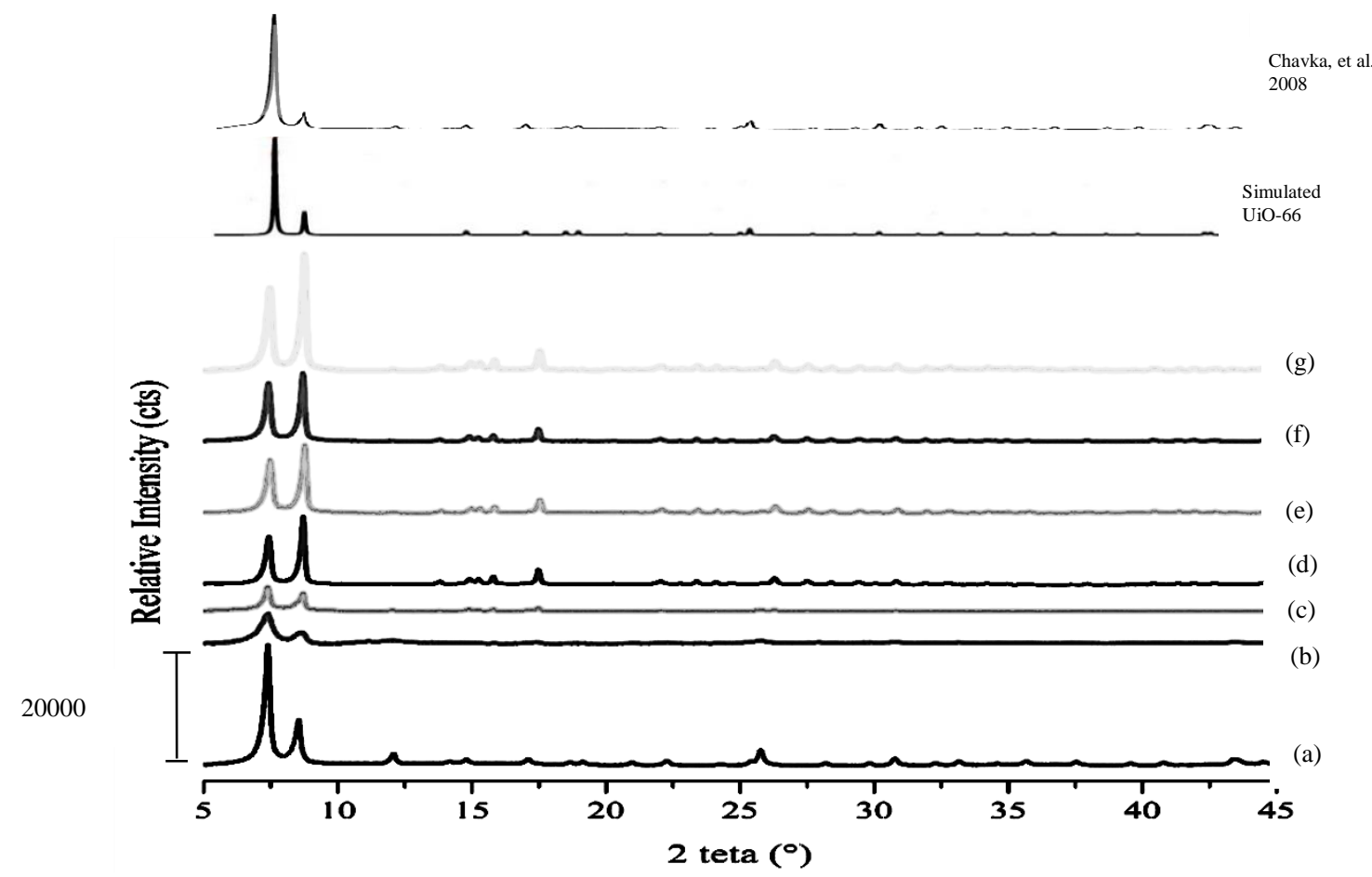

Figure 2. X-ray diffractograms of UiO-66 synthesized at $140^{\circ} \mathrm{C}$ with increasing time synthesis (a) $6 \mathrm{~h}$, (b) $12 \mathrm{~h}$, (c) $24 \mathrm{~h}$, (d) $36 \mathrm{~h}$, (e) $48 \mathrm{~h}$, (f) $72 \mathrm{~h}$, (g) 144h, reference UiO-66, and UiO-66 simulated

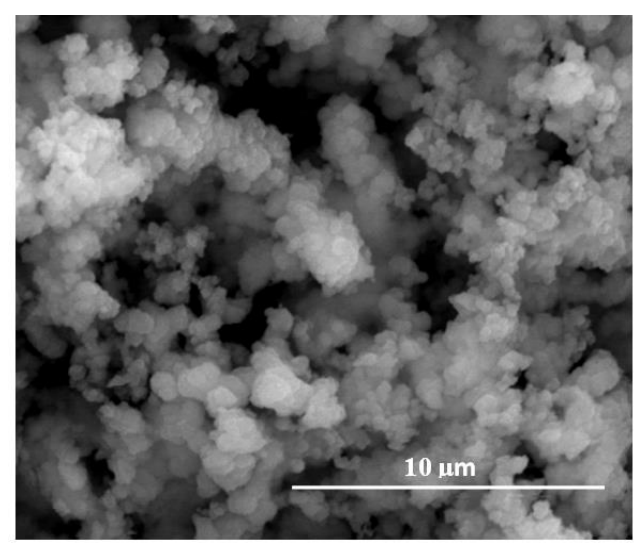

(a)

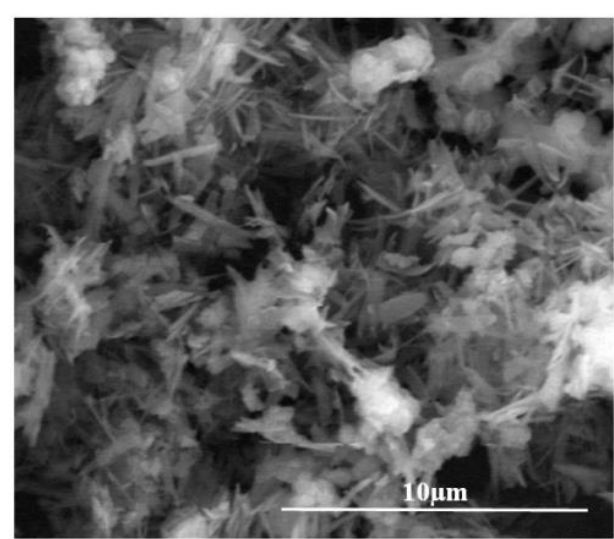

(b)

Figure 3. SEM picture of UiO-66 $140^{\circ} \mathrm{C}$, (a) $6 \mathrm{~h}$ and (b) $72 \mathrm{~h}$ 

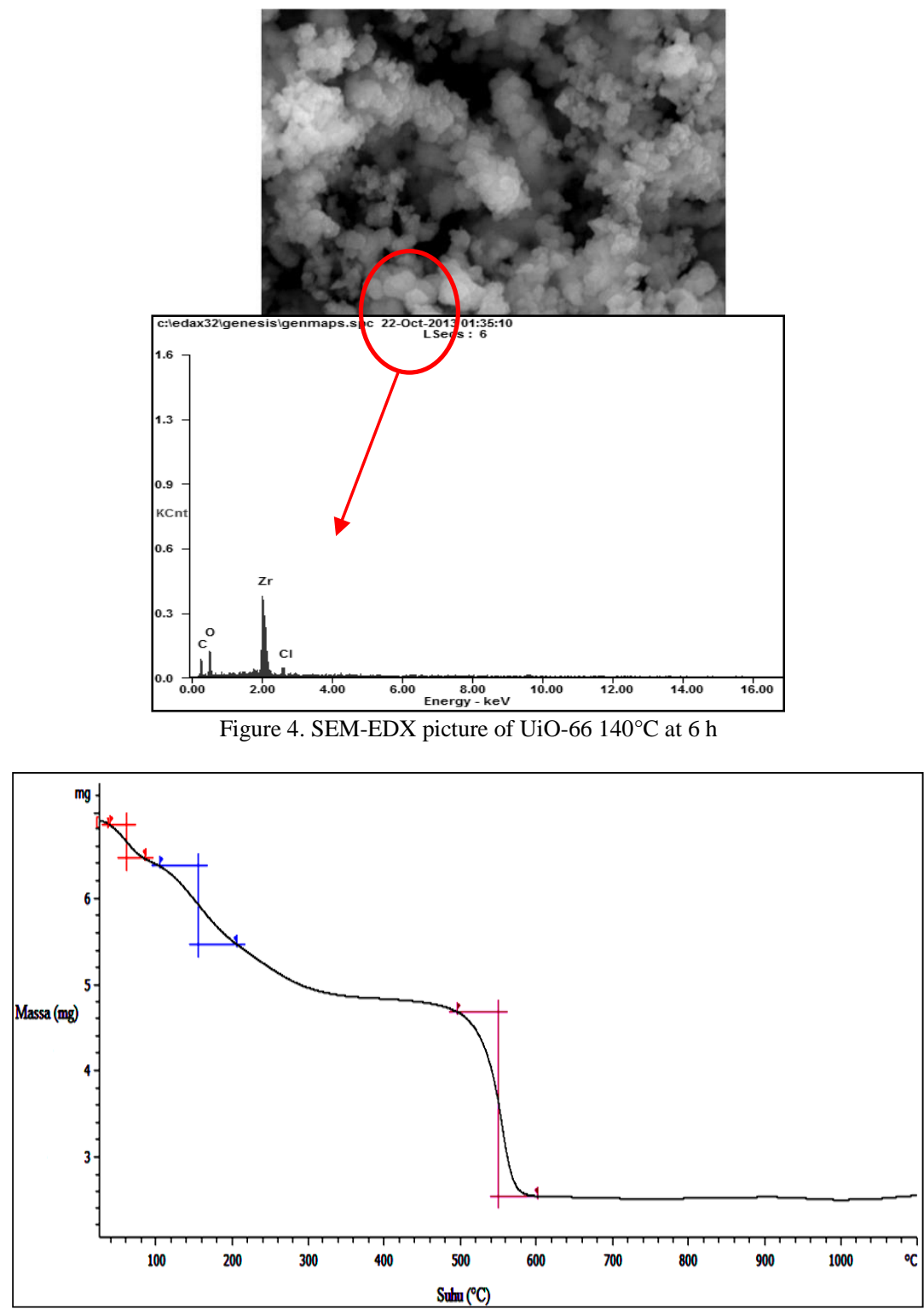

Figure 5. TGA Profiles of UiO-66 (Zr-MOF)

TABLE 1.

RELATIVE CRYSTALLINITY OF ZIF-8 CRYSTALS SYNTHESIZED WITH VARIATION OF HEATING TIME

\begin{tabular}{|c|c|c|c|}
\hline $\begin{array}{l}\text { Heating time } \\
\text { (h) }\end{array}$ & $2 \theta\left({ }^{\circ}\right)$ & $\begin{array}{c}\text { total area under the } \\
\text { curve }\end{array}$ & Kriatalinitas (\%) \\
\hline 6 jam & $\begin{array}{l}7,39 ; \\
8,53 ; \\
25,76\end{array}$ & 2676 & 100 \\
\hline $12 \mathrm{jam}$ & $\begin{array}{l}7,39 \\
8,54 \\
25,78\end{array}$ & 5598 & 44,2 \\
\hline 24 jam & $\begin{array}{l}7,38 \\
8,73 ; \\
25,79\end{array}$ & 3990 & 31,5 \\
\hline 36 jam & $\begin{array}{l}7,43 \\
8,72 \\
24,71\end{array}$ & 6957 & 54,8 \\
\hline 48 jam & $\begin{array}{l}7,48 \\
8,78 \\
25,84\end{array}$ & 8492 & 66,9 \\
\hline 72 jam & $\begin{array}{l}7,41 \\
8,72 \\
25,79\end{array}$ & 7986 & 63,0 \\
\hline $144 \mathrm{jam}$ & $\begin{array}{l}7,47 \\
8,77 ; \\
25,80\end{array}$ & 12109 & 95,5 \\
\hline
\end{tabular}

